\title{
Estrategias de gestión de proveedores en la industria minera cuprífera de Chile
}

\author{
Culverwell, Malaika * \\ Lufin Varas, Marcelo ** \\ Romani Chocce, Gianni ***
}

\section{Resumen}

Dentro de la función abastecimiento y adquisiciones de las compañías mineras en general, existe un reconocimiento explícito de que una compañía de explotación minera no puede sostener su competitividad y éxito en un mercado global sin la ayuda de sus proveedores. Como consecuencia de esta apreciación, varias de las compañias mineras más grandes de Chile y de América Latina han emprendido estrategias de administración de proveedores (Supply Base Management o SBM). En general, la literatura de SBM discute varias etapas en el proceso de desarrollo de una base de proveedores de clase mundial. No obstante esta evidencla teórica, se percibe un déficit significativo en la literatura con respecto a la perspectiva del proveedor y a la forma en que es integrado en el proceso de SBM, cuestión también conocida como selección de proveedores. En ese contexto, el objetivo de este trabajo es analizar la relación entre las empresas mineras y sus proveedores locales (en el país), desde la perspectiva del proveedor. Con tal fin, fue realizada una investigación empírica con cuestionario estructurado de preguntas abiertas y cerradas a los diferentes proveedores de las empresas mineras. Los resultados muestran que la perspectiva del proveedor puede revelar información significativa con respecto al nivel del flujo de información existente en la relación comprador-proveedor, sobre flexibilidad del proveedor para adaptarse unilateralmente a los cambios contractuales y la dependencla del proveedor a los compradores. Esta información proporciona un input de información valioso para el proceso de decisiones asociado a la implementación de una estrategla SBM y en particular en la evaluación y selección del proveedor.

Palabras clave: Abastecimiento, administración de proveedores, industria minera, desarrollo regional, subcontratación.

\section{Recibido: 01-04-24. Aceptado: 01-07-27}

* PhD. en Administración, Judge Institute of Management Studies, University of Cambridge. Profesora visitante e investigadora asociada de IDEAF. Avenida Angamos 0610, Antofagasta, Chile.

* M.A. en Economía. ILADES-Georgetown University. Académico del Departamento de Economia, Universidad Católica del Norte y Director de IDEAR. E-mall: mlutin@ucn.cl

*** Dra. en Administración, Universidad de Sao Paulo. Académica del Departamento de Clencias Empresariales, Universidad Católica del Norte e investigadora asociada de IDEAR. E-mail: gachocce ucn.cl 


\title{
Supplier Management Strategies in the Chilean Copper Mining Industry
}

\begin{abstract}
In reference to supply and purchasing functions in mining companies in general, there is explicit recognition on the part of these companies that they cannot maintain competitiveness and succeed in global markets without help from their suppliers. As a result of this, several of the larger mining companies in Chile and Latin America have implemented supply base management (SBM) strategies. SBM literature covers various stages in the development of a base of worid class suppliers. However, even in the face of theoretical evidence, there is a lack of significant literature as to the perspectives of the supplier and the manner in which his perspectives can be integrated into the SBM process. This is also referred to as supplier selection. The objective of this paper is to analyze the relationship between mining companies and their local suppliers, from the supplier's perspective This empirical research was based on the development of a structured questionnaire with controlled and open answers which was applied to mining company suppliers. The results show that the suppliers' viewpoint can offer significant information concerning the level and flow of intormation, supplier flexibility to adapt to unilateral contractual changes, and supplier dependency on buyers. This intomation provides valuable input for the decision making process related to the implementation of SBM strategies particularly in the evaluation and selection of suppliers.
\end{abstract}

Key words: Supply, supply management, mining industry, regional development, sub-contracting.

\section{Introducción}

Engeneral las firmas, en el proceso de concentrarse en las áreas estratégicas de sus negocios, llegan a ser más dependientes de sus proveedores para lograr producir oportunamente productos y servicios de calidad a precios competitivos.

Con anterioridad, la colaboración con proveedores o programas de desarrollo de proveedores eran establecidos sobre la base de otras prácticas de administración de proveedores, tales como la evaluación y certificación de proveedores y la racionalización de la base de oferentes (Hahn et al., 1990). Desde una perspectiva estratégica, la firma proveedora que se compromete en una práctica

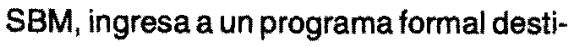

nado a incrementar su desempeño y capacidades en todas las áreas requeridas por su empresa cliente.

\section{Antecedentes bibliográficos}

Krause y Handfield (1999), Watts y Hahn (1993) y Monczka et al. (1993) han definido varios modelos del proceso y en todos ellos se bosquejan varios estados en el desarrollo de una SBM. El elemento común en todos estos estudios es que el estado inicial es esencialmente un esfuerzo unilateral desde la firma cliente para evaluar su propia base de proveedores sin que exista una significativa adhesión de las firmas proveedoras.

La participación de los proveedores en la estrategia SBM, se asume en un estado posterior y puede ser visualizada 
desde la gerencia como un aporte al desarrollo de la firma, por cuanto su adhesión puede generar negocios importantes y producir mayor información de las firmas que podrian reforzar las actitudes de asistencia y de colaboración entre las empresas proveedoras y compradoras.

\subsection{Estrategia de administración de proveedores (SBM) y desarrollo de proveedores}

La intensidad de la competencia en el ambiente donde se desenvuelven las empresas en la actualidad ha incentivado la generación de alianzas. En este contexto, las redes y la administración de las cadenas de proveedores son vehículos mediante los cuales las firmas pueden generar ventajas competitivas. En el esfuerzo por asegurar fuentes competentes de abastecimiento, que provean un flujo ininterrumpido de insumos y materiales a precios razonables, la función de adquisiciones de las empresas requiere el desarrollo de un conjunto de actividades que pueden ser genéricamente definidas como "administración de proveedores". Estas labores implican en primer lugar, la selección de proveedores (SS) competentes desde una perspectiva tecnológica, de calidad, considerando plazos de entrega y costos. En una etapa posterior, estas relaciones se profundizan requiriéndose trabajar junto con los proveedores para incrementar y mejorar sus capacidades (Hahn et al., 1990), esta nueva dimensión es sinónimo de "Desarrollo de Proveedores" o "Supplier Development" (SD).

Las actividades incluidas entonces en la estrategia de SD son: introducir competencia en la base de proveedores de la firma, aplicar sistemas de evaluación de proveedores, introducir aumentos en las expectativas de desempeño e implementación de acciones de reconocimiento y premiación, además de generar promesas de beneficios futuros, entrenamiento y capacitación del personal de los proveedores, complementadas con actividades de intercambio y pasantlas de trabajadores de firmas proveedoras $y$ clientes y, finalmente, la colaboración e inversión directa de la firma cliente en la firma proveedora (Monczka y Trent, 1991).

No obstante lo interesante de la fase de desarrollo de proveedores, el énfasis de este trabajo se ha puesto en la fase de selección de proveedores, en la cual el acento está puesto en incrementar el desempeño exhibido por el proveedor, tal como lo señala Hahn, Watts y Kim (1990) el programa SS puede ser visto como una fase de implementación del proceso completo.

Esta fase inicial de selección ha sido claramente delineada y descrita por Krause and Handfield (1999). Según estos autores, la organización de la función de adquisiciones requiere la especificación de las necesidades de incremento en el desempeño del proveedor y establecer metas cuantitativas de sus niveles de logro exigidos. Estas necesidades inicialmente conciernen sólo a la base corriente de proveedores y luego podrán irse aplicando a la totalidad de las funciones de compra.

En el Gráfico 1 se aprecia el modelo conceptual del proceso de decisiones en una estrategia de administración de proveedores. 
Proveedores en la industria minera de Chile

Culverwell, M.; Lufin V., M. y Romani C., G.

Gráfico 1

Modelo Conceptual sobre el Proceso de Decisiones de Selección de Proveedores en una Estrategia SBM

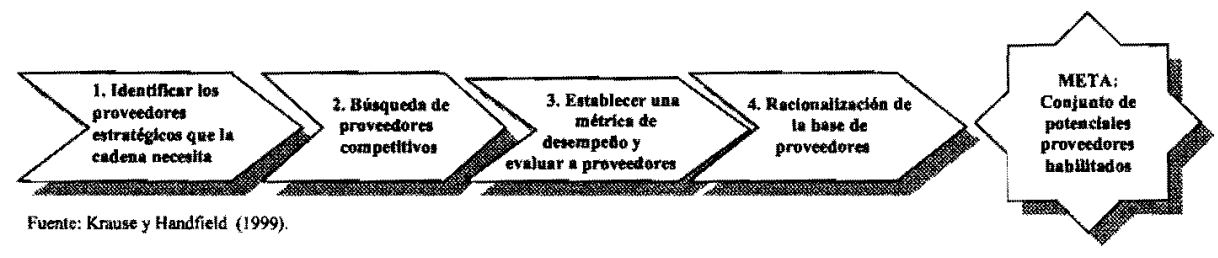

Cuando la firma cliente no está satisfecha con los niveles corrientes de desempeño de sus proveedores, o cuando reconoce la necesidad de incrementar estos niveles, ella puede iniciar una estrategia de selección de proveedores. Adicionalmente, si la firma tiene nuevos requerimientos y estos no pueden ser satisfechos por sus actuales proveedores, debería comenzar un proceso de búsqueda y selección de nuevos oferentes. En ambos casos, la evaluación de los proveedores facilitará la selección y permitirá identificar la áreas que deben ser fortalecidas en una relación más permanente (Hahn et al., 1990).

En la discusión sobre qué elementos permiten una evaluación más comprensiva de los proveedores, debe considerarse ampliar los cánones tradicionales de valoración (calidad, oportunidad, precio, etc.) incorporando la noción de nivel de capacidad y calidad de participación.

La estimación del nivel de capacidad del proveedor ha sido abordada empíricamente en el estudio de Watts y Hahn (1993). Ellos muestran la importancia de ciertas clases de capacidades en los proveedores. En las 81 compañías consideradas en el estudio, siempre se mencionan los mismos elementos priorizados en similar orden, el estudio concluye que los compradores están más preocupados del corto plazo, tratando de mejorar el producto para reducir los costos de entrega que de los aspectos de largo plazo, como son capacidades asociadas a sistemas o procesos productivos que asegurarian reducciones de costos futuras.

Siguiendo esta preocupación por completar una evaluación comprehensiva de los proveedores, Krause $y$ Handfield (1999) notan que las más comunes deficiencias en una base de proveedores radica en errores en áreas como: costos, calidad, entrega y capacidades tecnológicas y destacan que otro problema común con los proveedores es la falta de destrezas administrativas, mala salud financiera y en la imposibilidad de expandir sus capacidades.

No obstante, los avances en el proceso reseñado en la literatura sobre evaluación de proveedores o selección de proveedores normalmente no se discute la posibilidad de integrar la perspectiva del proveedor, cuya participación efectivamente contribuiría a lograr una comprensión mayor. En este contexto, la "Perspectiva del Proveedor" podría implicar una comunicación regular con los pro- 
veedores para comprobar si ellos están concientes de los cambios en los métodos de evaluación, la opinión de los oferentes sobre sus capacidades actuales y futuras, y sobre el tipo de asistencia que ellos requieren para lograr los niveles requeridos, asi como de quienes ellos preferirian proviniera esa ayuda.

\subsection{Importancia de la "Perspectiva del Proveedor"}

Centrándose en la relación contractual entre proveedores-demandantes en la industria minera, las autoridades preocupadas del desarrollo en los paises en desarrollo sienten que las grandes empresas tienen una responsabilidad en sostener a las pequeñas y medianas empresas de las economías locales. De acuerdo con Halbach (1989) la positiva contribución de la subcontratación para la economía de manera general descansa en el efecto estructural de un sector industrial que se complementa a sí mismo en forma óptima en términos de tamaño, la transferencia de tecnología y la creación de destrezas tecnológicas que se desprenden de los encadenamientos interindustriales, además de la utilización de la fuerza de trabajo y el capital.
Sin embargo, la realidad de la industria minera desde hace décadas es que la producción es fuertemente intensiva en capital y emplea directamente una baja proporción de la fuerza de trabajo nacional y regional, con importantes salidas de capital como retorno a las fuentes financieras extranjeras ${ }^{1}$. De esta forma, la minería podría considerarse como una "industria enclave" con modestos encadenamientos locales de producción (ver Tabla $N^{\circ} 1$ ). Esto significaría una baja retención local de excedentes (Auty, 1998). Por otra parte, como los flujos de inversión extranjera directa de las compañias mineras y de otras fuentes de capital extranjero han llegado a ser aceptadas como una importante fuente para el crecimiento, los encadenamientos industriales hacia atrás son también apreciados como vehiculos que permiten el cumplimiento de mutuos intereses entre el capital extranjero y las economías locales (Yotopoulos y Nugent, 1976).

\section{Modelo conceptual}

Una preocupación importante en las autoridades políticas en general, ha sido como incrementar el tamaño de la economía regional a través de profundi-

1 En un original trabajo publicado en 1950, H.W. Singer criticó la inversión extranjera directa (IED) en los países menos desarrollados porque tendian a concentrarse en industrias extractivas con bajos encadenamientos productivos hacia adelante o hacia atrás. Debido a la falta de "efectos de multiplicador secundario" en el país receptor de la IED, ésta podia ser vista como generadora de enclaves productivos de la economía más desarrollada destinados a proveer materias primas a la industria extranjera. Solamente la inversión extranjera en industrias manufactureras podría proveer "un estimulo para otras industrias a través de encadenamientos que aumentarán el conocimiento técnico, la educación urbana y el dinamismo asociado a una civilización urbana-industrial". 


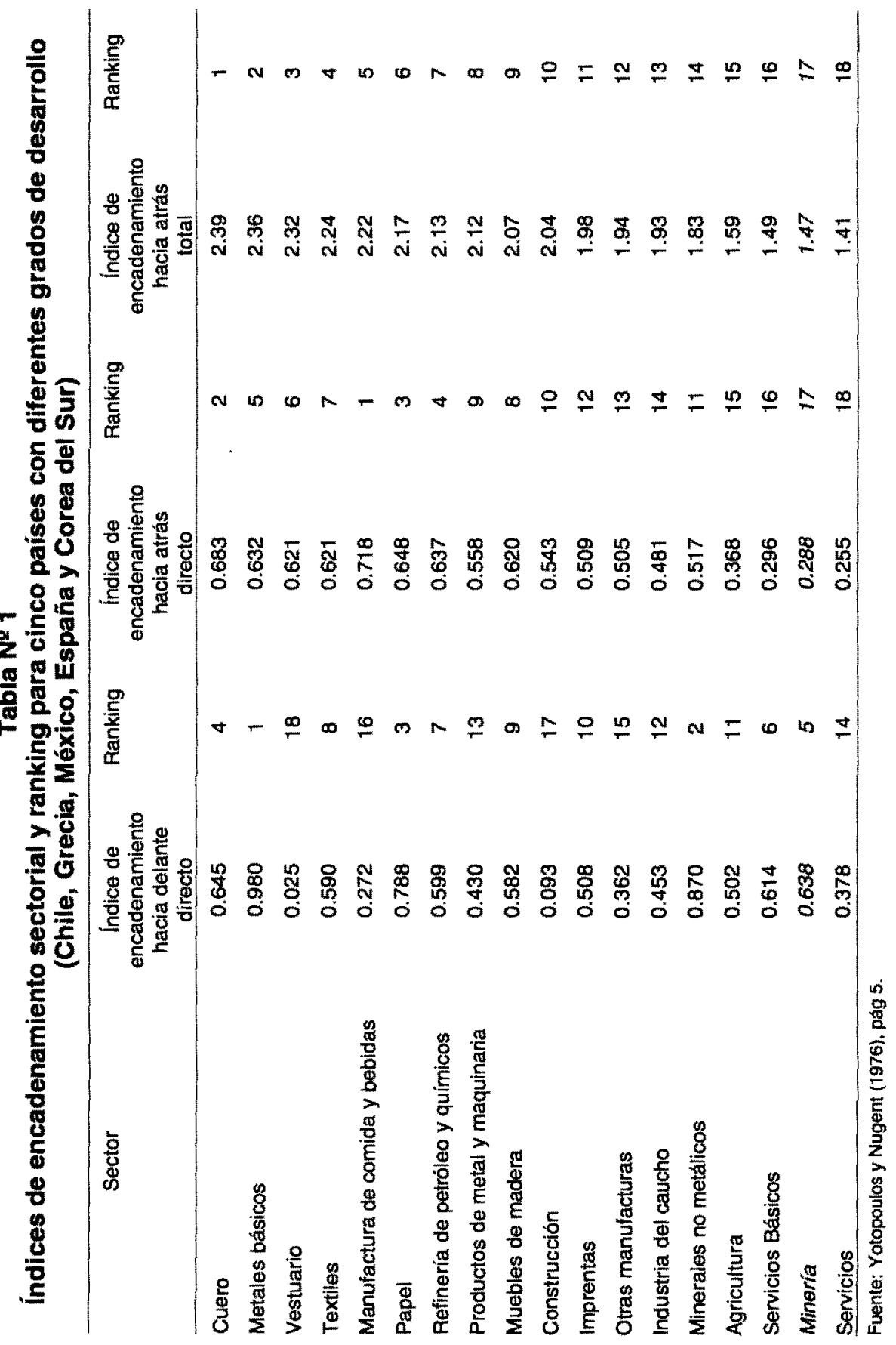


zar la eficiencia productiva o ganancias de competitividad (Battat et al., 1996). Un camino para lograr este propósito es preocuparse de las necesidades de los proveedores locales, entre los que destacan las formas de relaciones contractuales, de manera de lograr mayor seguridad en que los contratos que se gana puedan ser mantenidos e incluso ampliados, generándose transferencia de tecnologías (hard y soft) como resultado de la interacción con las industrias mineras y el desarrollo de habilidades tecnológicas y capacidades financieras en las firmas.

El Gráfico 2 muestra el Modelo Conceptual de la Significación de la Integración de los Proveedores en una Estrategia SBM que se ha aplicado para definir e interpretar las vinculaciones de las firmas en los casos regionales en estudio.

Para el diseño de la investigación se consideró el hecho que desde una perspectiva más amplia la estrategia de SBM también tiene fuertes implicancias en el desarrollo de encadenamientos ha- cia atrás, lo cual pudiera ser especialmente interesante en los paises menos desarrollados, en los cuales las grandes corporaciones y los disenadores de politica están tratando de encontrar formas para fortalecer las relaciones clientesproveedory a la industria de la subcontratación en general. Esto es significativamente importante desde la perspectiva de la modernización y aumento de la idoneidad de las pequeñas y medianas empresas quienes enfrentan el desafío de mejorar su competitividad doméstica y global tratando de mantener o incrementar esta clase de contratos y vinculaciones.

\section{Metodología de trabajo}

Como el objetivo del trabajo es establecer la importancia de considerar la perspectiva del proveedor para el desarrollo de una estrategia de SBM, particularmente la forma en que las estrategias de evaluación de proveedores implemen-

\section{Gráfico 2}

\section{Modelo Conceptual SBM Aplicado}

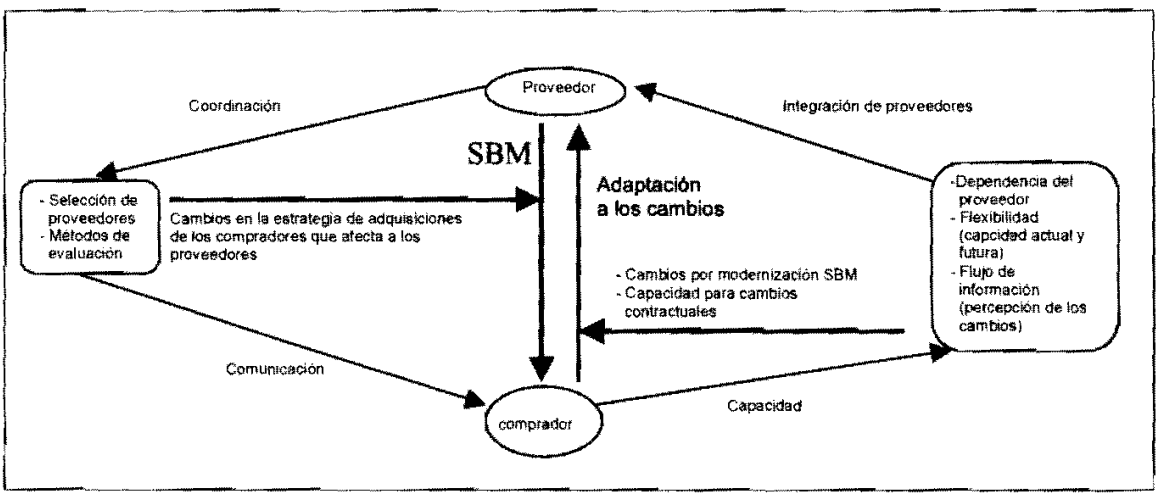

Fuente: víaboración propia. 
tadas por las firmas clientes están afectando los negocios y cómo estas relaciones contractuales pueden ser mejoradas. Se utilizó el estudio de casos vinculados a cinco compañías mineras localizadas en la II Región-Autofagasta-, privilegiando la descripción de la situación actual y esperada, cubriendo así un horizonte temporal desde 1997 al 2003 aproximadamente.

Para obtener la caracterización de las estrategias de los proveedores y de las prácticas asociadas con las estrategias SBM se utilizaron entrevistas y encuestas.

La información recogida por las entrevistas en profundidad fue grabada; para su vaciado se utilizó un formato estructurado previamente; todas estas actividades se extendieron por cerca de un año.
Finalmente para complementar la información de las entrevistas se utilizaron los reportes públicos de las empresas, como memorias y balances, además de los informes de los respectivos departamentos de contratos.

La base de datos de los proveedores contenía un universo total de 639 firmas, localizadas a lo largo de todo Chile (Gráfico 3). Se decidió restringir la muestra a aquellas que tuvieran, desde la perspectiva de las empresas clientes, una mayor importancia.

Utilizando este criterio, se seleccionó a $\mathbf{2 0 0}$ firmas con fuertes vinculos con las empresas clientes radicadas en la zona. Finalmente, el número total de empresas que respondió la encuesta fue de 135 representando el $60 \%$ de la muestra inicial.

\section{Gráfico 3}

\section{Empresas mineras clientes}

MAYORES MINAS DE COBRE EN EL MUNDO SEGÚN LOCALIZACION

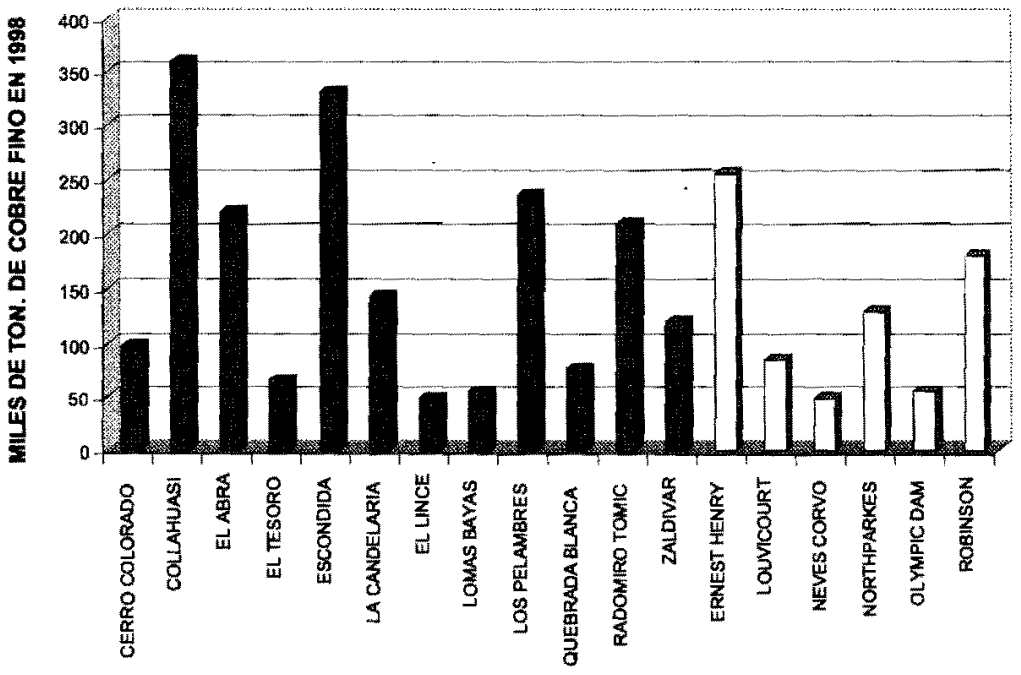

MINAS LOCALIZADAS EN CHILE (OSCURO) Y EN EL RESTO DEL MUNDO 
Las entrevistas estuvieron centradas en el personal de compra y de asistencia, pero también incluyeron al usuario final (ingenieros), de mantención, de finanzas y de control de calidad. Dado que alguna información fue de naturaleza confidencial se requirió de varios intentos para lograr acceso a los datos requeridos.

\subsection{Características de la muestra}

La industria minera fue elegida como toco de este trabajo por dos razones: primero, porque en esta industria se ha desarrollado un creciente interés por investigaciones destinadas a lograr avances sobre que tipo de arreglos deben ser promovidos en la gestión de abastecimiento y materiales del sector, lo que además se ha traducido en una práctica interesante dentro de las empresas en el área (Richardson, 1991), y segundo porque el sector minero está bajo una constante observación sobre cuál es el impacto socioeconómico y ambiental en la comunidades receptoras de esta clase de inversiones. Las autoridades politicas en particular están muy interesadas en aprender cómo los proveedores locales son afectados por las estrategias de abastecimiento de sus clientes transnacionales (Battat $\theta$ al., 1996).

Finalmente se usó la industria cuprifera en Chile, porque en este sector es posible analizar las interacciones entre grandes compañías transnacionales $y$ nacionales, con sus proveedores en el contexto de un país en desarrollo ${ }^{2}$.

El análisis se centró en las empresas vinculadas con las 5 compañias mineras más relevantes: División Chuquicamata de CODELCO, División Radomiro Tomic de CODELCO, Minera Escondia Ltda, Sociedad Contractual de El Abra y Compañía Minera Zaldivar, quienes en 1998 produjeron el $80 \%$ o US\$3.245 millones de las exportaciones regionales de cobre. Estas empresas también están en la lista de las mayores productoras en el mundo.

La muestra de firmas proveedoras consistió en 135 compañías de diferentes tamaños (según promedio de ventas anuales) y de diversas actividades (ver Tabla $\left.N^{2} 2\right)$, la mayoría de estos oferentes tienen un tamaño grande o medio y el 61 $\%$ del total tiene su casa matriz o una sucursal principal en la 11 región -Autofagasta-. La muestra fue seleccionada de las bases de datos de los organismos de desarrollo de PYMES y de los listados de las grandes empresas demandantes ya reseñadas. Casi todas las empresas de la muestra tienen importantes vinculaciones con las empresas compradoras concentrando en ellas cerca del $50 \%$ de sus ventas anuales.

En el Gráfico 4 se representa gráficamente la información sobre las firmas nitud de sus operaciones y niveles de inversión en nuevos proyectos. En promedio la industria minera contribuye entre el 55 y $60 \%$ d el PIB regional y $8 \%$ del PIB nacional. 


\section{Tabla N 2}

\section{Actividades de las empresas de la muestra}

Actividades de las Empresas

Transporte Personal

Aseo industrial

Tronaduras

Casino

Sondaje

Seguridad industrial

Movimiento de Tierra

Fabricación Maquinaria y Equipo

Arriendo Equipos

Torneria

Soldaduria

Calderería

Fabricación con Metales

Ingeniería de Proyectos

Servicios Mecánicos

Reparación y Manutención Maquinaria

Otros Servicios

Fuente: Elaboración propla.

proveedoras, incluyendo distribución por ventas medias anuales, frecuencia según tipo de productos y/o servicios ofrecidos y frecuencia según clientes principales reconocidos.
Importancla Relativa ${ }^{3}$

0.0033

0.0066

0.0066

0.0099

0.0132

0.0132

0.0362

0.0395

0.0526

0.0592

0.0658

0.0691

0.0855

0.0855

0.0921

0.0954

0.2664

Las firmas mineras consideradas se encuentran dentro del grupo de las más importantes, de acuerdo a la importancia que tienen dentro del grupo de las empresas clientes relevantes, según un

3 El concepto de importancla relativa se define como una opinión dominante en la estructura de respuestas de las firmas, para efectos prácticos se definió de la siguiente forma:

Sea: $w_{k f}=1$ si la firma $i$ declara que está ofreciendo el servicio o producto $d$ a sus empresas clientes. $w_{i d}=0$ si la firma $i$ declara que no está ofreciendo el servicio o producto.

La importancia relativa del producto o servicio des: $\beta_{d}=\left[\frac{\sum_{i=1}^{1} w_{i d}}{\mid \sum_{d=1}^{D} \sum_{i=t}^{f} w_{i d}}\right\rceil$, donde $I=136$ empresas proveedoras y $D=17$ productos o servicios ofrecidos. 


\section{Gráfico 4}

Empresas proveedoras. Distribución de las firmas de la muestra según ventas anuales

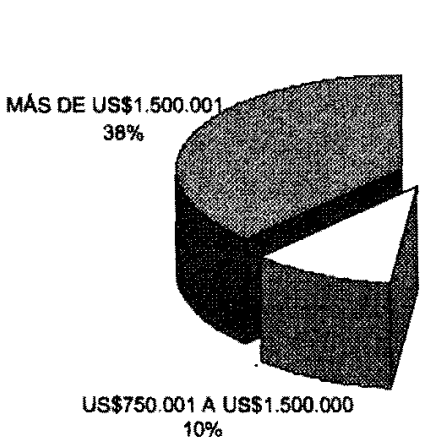
$10 \%$

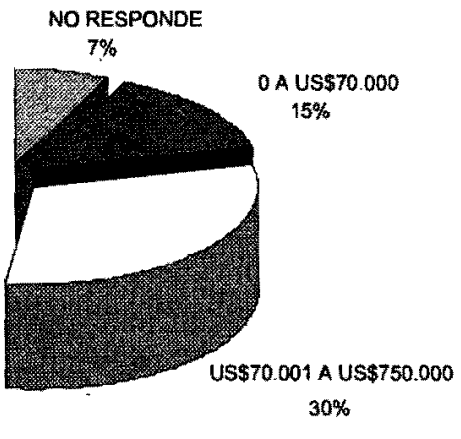

indicador de importancia relativa considerado en la Tabla № 3.

\subsection{Técnica de recolección de datos de proveedores}

Los datos sobre los proveedores fueron recolectados mediante una encuesta a ellos mismos, que fue dividida en tres partes: (i) una sección que solicitaba información general sobre la empresa, sus negocios y principales clientes; (ii) preguntas sobre cambios percibidos en la estrategia de adquisiciones de sus clientes y los efectos de ellos sobre la relación y (iii) preguntas especificas sobre que áreas de asistencia están o esperan requerir en el futuro para enfrentar los

\section{Tabla N 3}

Importancia de las firmas clientes

\begin{tabular}{lcc}
\hline Empresa & Frecuencia & Importancla Relativa \\
\hline Escondida & 101 & 0.0849 \\
Soquimich & 98 & 0.0824 \\
Chuquicam & 82 & 0.0689 \\
Lomas Bayas & 82 & 0.0689 \\
M.Blancos & 81 & 0.0681 \\
Zaldivar & 78 & 0.0655 \\
Michilla & 77 & 0.0647 \\
Refimex & 69 & 0.058 \\
R. Tomic & 64 & 0.0538 \\
El Abra & 63 & 0.0529 \\
Rayrock & 60 & 0.0504 \\
\hline
\end{tabular}

Fuente: Elaboración propia. 
cambios esperados y fuente de esta asistencia probable.

La encuesta fue construida con el propósito de maximizar la cobertura de los aspectos principales de las estrategias SBM y minimizar la no respuesta a causa de una garantía de confidencialidad de la información proporcionada.

\section{Resultados}

Los resultados de la investigación, para efectos de orden, se presentan de acuerdo a las siguientes preguntas de investigación que estructuraron el trabajo de campo.

1. ¿Cuáles son las percepciones de los proveedores sobre los cambios en la estrategia SBM de sus clientes?

2. ¿Cuáles son las percepciones de los proveedores sobre desafíos que resultan de estos cambios?

3. ¿Desde quéclase de agentes los proveedores esperan asistencia en el futuro para afrontar estos desafíos?

El análisis de los datos se concentró en responder las preguntas de investigación enunciadas, su examen detallado reveló conceptos que describen la relación contractual que mantienen los proveedores con sus clientes principales.
Los conceptos principales son los siguientes:

- Flujo de información desde el comprador al proveedor.

- Flexibilidad del proveedor para responder a los cambios en la relación.

- Dependencia del proveedor de los clientes principales o de otros actores de asistencia sectorial.

\subsection{Flujo de información}

Pregunta $N^{\circ} 1:$ ¿Cuáles son los cambios actuales y esperados en la estrategia de adquisiciones de las firmas clientes?

La información se representa en el Gráfico 5. En ella se define la frecuencia relativa ${ }^{4}$ de los aspectos más mencionados por las firmas entrevistadas, contraponiéndose las características de la estrategia presentes hoy con aquellas que se espera se agudicen más en un horizonte de 3 años en el futuro.

Los resultados mostrados en el gráfico, revelan que las firmas proveedoras han percibido la creciente importancia relativa de la evaluación de los sistemas de seguridad (columna 8), la utilización de internet (columna 6), la calidad de servicio o producto (columna 3 ), el impacto

4 Sea: $q_{\text {ij }}=1$ si la firma iestima que la estrategia j está siendo (o será) utilizada por sus empresas clientes. $q_{y}=0$ si la firma iestima que la estrategia $j$ no está siendo (o no será) utilizada por sus empresas clientes.

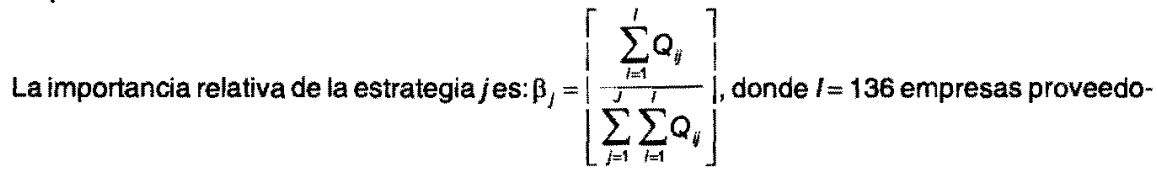
ras $y J=10$ estrategias posibles. 


\section{Gráfico 5}

Percepciones sobre estrategias de adquisiciones

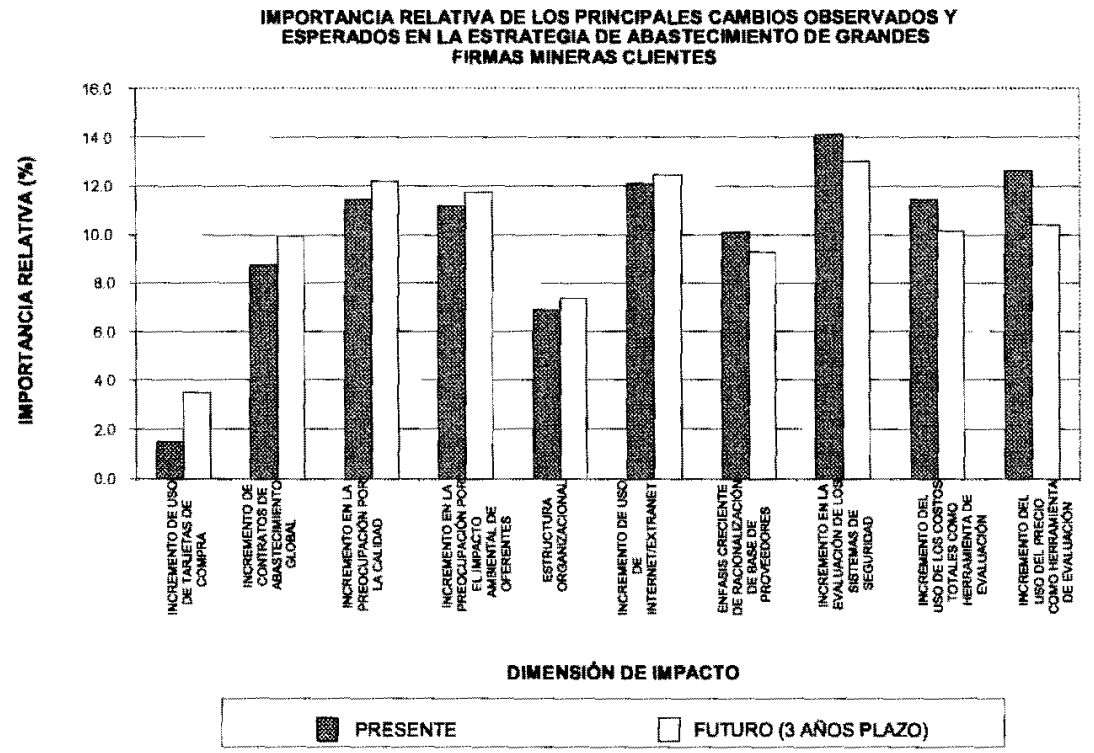

ambiental (columna 4) y la aplicación en general de métodos de evaluación (columnas 9 y 10). Del mismo modo no parecen bien informados sobre otras prácticas que pudieran impactar profundamente la forma actual de las relaciones; por ejemplo, se percibe una baja en la importancia del ítem de costos y precios en la estrategia de evaluación futura, pero sin que ello se asocie al incremento importante de los aspectos de calidad o seguridad en la misma evaluación.
En la Tabla № 4, se presenta un resumen de los resultados de la misma pregunta, pero conteniendo las opiniones a las empresas mineras clientes.

Otro punto de interés es definir qué aspectos particulares de las acciones de las empresas proveedoras se han visto más afectadas por la dinámica de las estrategias de abastecimiento de sus firmas clientes. En el Gráfico 6 se muestra la frecuencia absoluta ${ }^{5}$ (tamaño de las barras) de las respuestas de las empresas sobre

5 Sea: $P_{\text {ik }}=1$ si la firma iestima que la estrategia tipo $\mathrm{k}$ de las firmas clientes afecta a algunas de los $s$ tipos de relaciones que la firma imantiene con diferentes actores del sistema productivo. $P_{I k}=0$ si la firma iestima que la estrategia tipo $k$ de las firmas clientes no afecta a algunas de los $s$ tipos de relaciones que la firma $i$ mantiene.

La importancia absoluta de la estrategia kes: $\alpha_{k}=\sum_{i=1}^{l} P_{k}$, donde $I=136$ empresas proveedoras. 


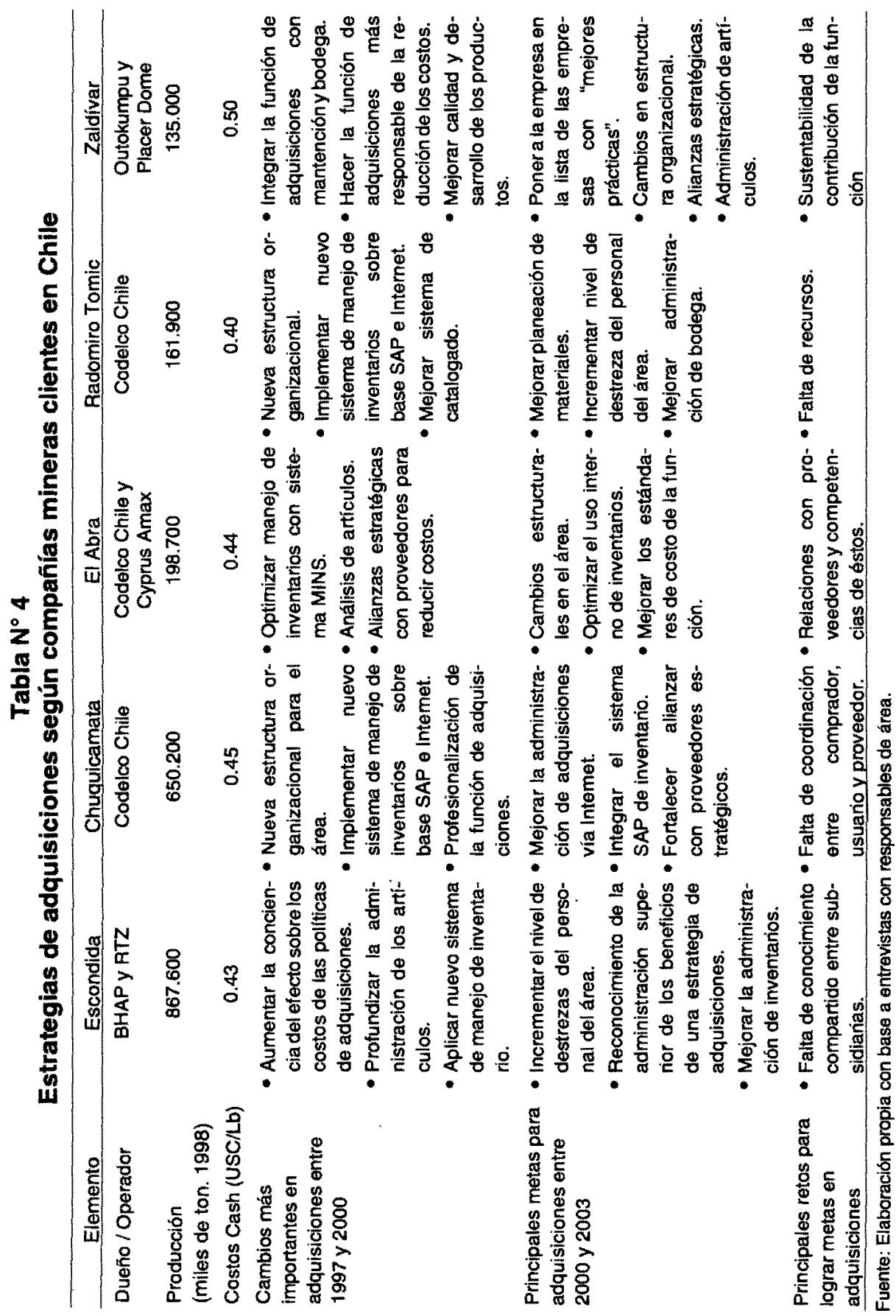




\section{Gráfico 6}

Tipo de relación de firmas proveedoras más afectadas según tipos de cambio en la estrategia de adquisición de empresa minera-cliente

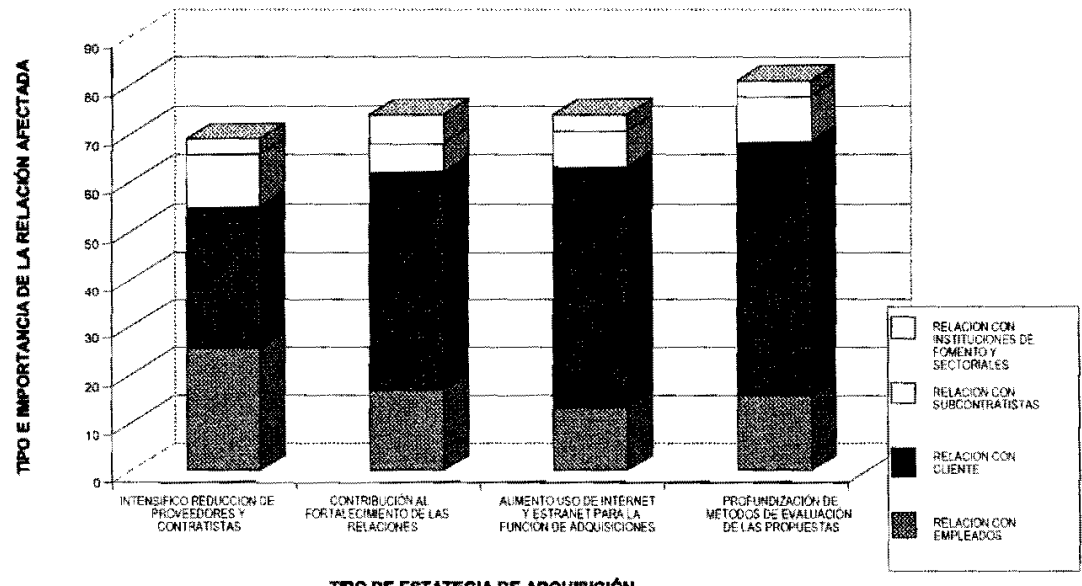

la importancia que atribuyen a los diferentes tipos de estrategias de adquisición implementadas. En él, destaca levemente la importancia de las prácticas de profundización de los métodos de evaluación aplicados al examen de las propuestas (columna 4).

Desde otra perspectiva, la estructura interna de cada barra en el gráfico muestra la importancia relativa ${ }^{6}$ de las diferentes relaciones de las firmas proveedoras que se han visto afectadas por las estrategias de las firmas clientes.
La base de las columnas representa el efecto que la respectiva estrategia de las firmas clientes ha tenido sobre la relación de las proveedoras con sus empleados.

Esta dimensión, adicionalmente, muestra un impacto mayor en la estrategia de reducción de proveedores y contratistas, probablemente por la natural sensibilidad de la fuerza de trabajo a esta clase de prácticas, y gradualmente va perdiendo importancia a medida que la atención se desplaza hacia estrategias más intensivas en el fortalecimiento de

6 Sea: $p_{i s}^{k}=1$ si la firma iestima que la estrategia tipo $k$ de las firmas clientes afecta su relación tipo $s$ particular, $p_{t^{*}}^{k}=0$ si la firma lestima que la estrategia tipo $k$ de las firmas clientes no afecta su relación tipo s particular. La participación relativa de cada relación s afectada dentro de la importancia absoluta de la estrategia kes: $\gamma_{k}^{s}=\left[\frac{\sum_{i=1}^{1} p_{i s}^{k}}{\sum_{s=1}^{s} \sum_{i=1}^{1} p_{k s}^{k}} \mid * \alpha_{k}\right.$, donde $t=136$ empresas proveedoras, $K=4$ estrategias posibles y $S=4$ relaciones. 
las relaciones interfirmas o la tecnologización de las mismas. Otra área que aparece intensamente afectada es la relación misma con el cliente, teniendo una importancia relativa mayor en los casos en que se han aplicado estrategias tecnologizantes (columnas 3 y 4 ).

Estos patrones revelan la profunda sensibilidad del quehacer de las firmas proveedoras a las estrategias de adquisición implementadas por las firmas clientes, lo que puede originarse en el poder monopsónico que subyace en la relación.

\subsection{Flexibilidad de los proveedores}

Pregunta № 2: ¿Cuáles son las percepciones de los proveedores sobre desafíos que resultan de estos cambios y como ellos se han preparado para responderlos?

La información recogida frente a la temática subraya la importancia que las firmas otorgan a su propia iniciativa y recursos; en este sentido puede entenderse que la forma más natural y reactiva de preparación consiste en apelar unilateralmente a la propia capacidad de adapta. ción de las empresas.
En el Gráfico 7 se representa la respuesta a cómo prepararse para asumír los retos planteados por sus firmas clientes. La respuesta dominante $(45 \%)$ corresponde al esfuerzo unilateral de las empresas proveedoras por tratar de mejorar su relación con la firma cliente. Con menor incidencia se reconoce la posibilidad de recurrir a instituciones de apoyo y fomento del sector, con el objeto de encontrar asistencia financiera y tecnológica.

Finalmente, sólo un grupo minoritario de empresas señala que la diversificación de los negocios puede ser la respuesta apropiada para enfrentar el desafí; queda pendiente en este grupo saber si se trata de una conversión de la actividad o simplemente una ampliación de la línea de productos y servicios ofrecidos.

La declaración de estos medios de desarrollo puede ser completada con un examen de los diferentes requerimientos de asistencia que las empresas declaran haber usado, tanto para asistencia técnica como de gestión. Estos requerimientos pueden ordenarse de acuerdo a la fuente de asistencia para la situación presente, que incluye la síntesis de los pasados 3 años.

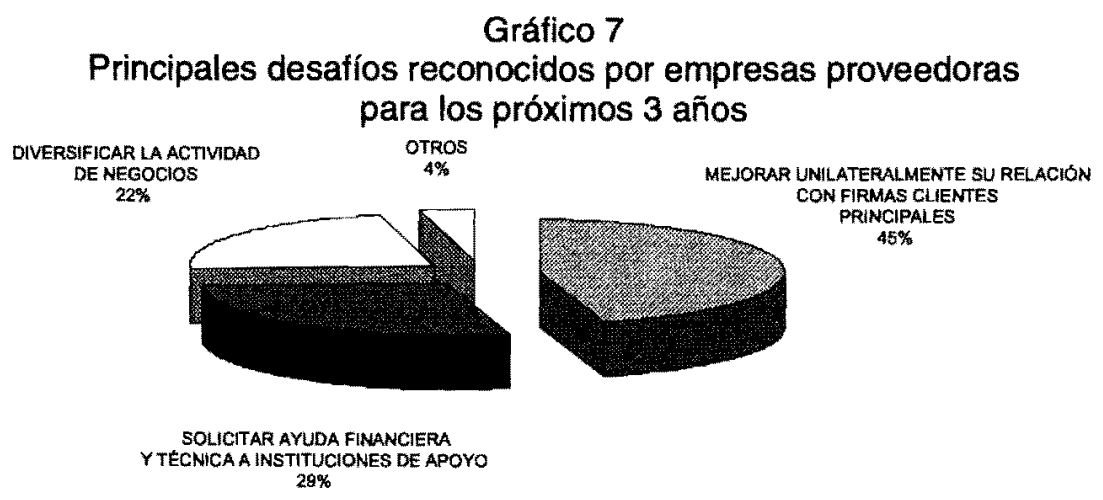




\section{Tabla N² 5}

\section{Importancia de las áreas de asistencia ${ }^{7}$}

Área de Asistencia Técnica

Presente

Proceso Productivo y Control de Calidad

Provisión de Equipos Computacionales

Provisión de Herramientas y Maquinaria

0.17

Compra de Materia Prima

0.14

Otros Aspectos

0.06

Área de Asistencia de Gestión

Contabilidad

Gerencia Administrativa y Personal

Marketing

0.17

Créditos a Plazos Convenientes

Préstamos No Reembolsables

0.08

Ayuda Financiera para 1\&D

0.09

Otros 0.01

Fuente: Elaboración propia.

En el conjunto denominado Tabla $N^{\circ} 5$ se muestra un indicador de importancia de las áreas de asistencia técnica y de gestión.

En general, la importancia de las áreas de asistencia técnica tiende a ser más alta en los casos de procesos productivos y control de calidad, y en la provisión de equipos computacionales. En el caso de la asistencia en gestión, es notable la mayor importancia otorgada al área contable, en contraste con los préstamos no reembolsables y la ayuda financiera para investigación y desarrollo.

Estas áreas revelarían la preocupación central por reforzar el control interno de la empresa desde una dimensión productiva y de proceso.

7 Para definir la importancia de las áreas de asistencia ya sea en tiempo presente $t=1$ o futuro $t=2$ se usó la siguiente expresión: Sea: $R_{i h}=1$ si la firma iestima que el área de asistencia (técnica o de gestión) $h$ ha sido (o será) desarrollada al interior de la empresa. $R_{\mathrm{b}}=0$ si la firma lestima que el área de asistencia (técnica o de gestión) $h$ no ha sido (o no será) desarrollada.

La importancia absoluta del área $h$ es: $\alpha_{h}=\sum_{t=1}^{l} R_{i h}$, donde $l=136$ empresas proveedoras.

La importancia relativa de cada área $h$ de asistencia es: $\beta_{h}=\left[\frac{\alpha_{h}}{\mid \sum_{n=1}^{H} \alpha_{h}}\right]$, donde $H=5$ en caso de áreas de asistencia técnica y $H=7$ en casos de áreas de asistencia de gestión. 


\section{Gráfico 8}

Áreas de asistencia técnica desarrolladas según fuente de la ayuda percibida en los recientes 3 años

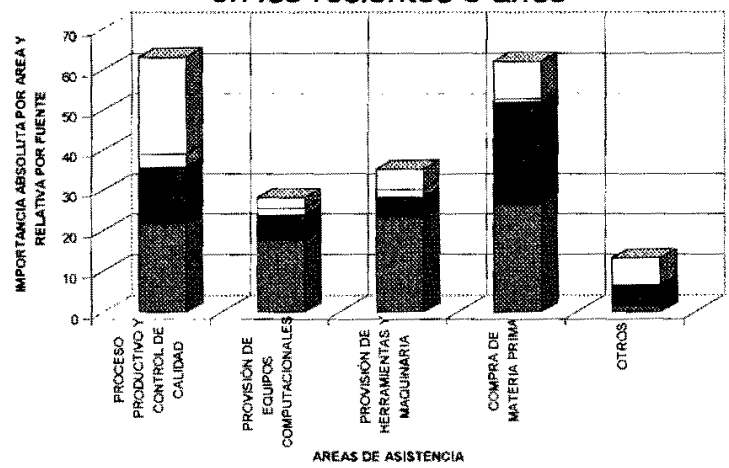

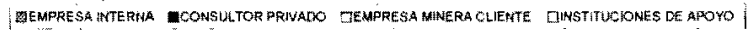

\section{Gráfico 9}

Áreas de asistencia de gestión desarrolladas según fuente de la ayuda percibida en los recientes 3 años

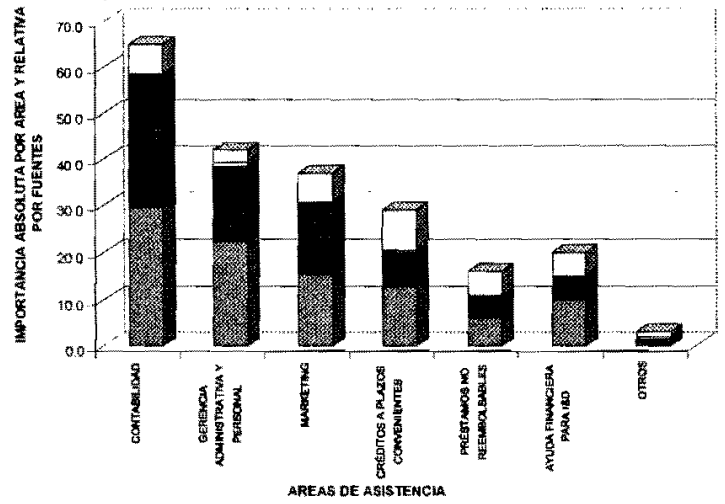

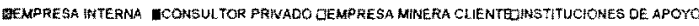

Finalmente las fuentes esperadas de asistencia ${ }^{8}$ se presentan por áreas, en los Gráficos 8 y 9 . Los dos gráficos seña- lan en primer lugar la comparación de las áreas prioritarias de asistencia técnica y de gestión, pero distinguiendo como han

8 Una fuente esperada de asistencia corresponde a cada uno de los actores sociales particulares con los cuales las empresas tiene vinculaciones, para efectos prácticos se definieron cuatro tipos: La Propia Empresa (internos), Consultores Privados, Empresas Mineras Clientes e Instituciones de Apoyo (Corfo, etc.). 
contribuido las diferentes fuentes potenciales de asistencia ${ }^{9}$.

En todas las áreas relevantes se aprecia una participación muy importante de la misma empresa, como fuente de asistencia, asi como la participación de consultores privados. Con relación a las instituciones de apoyo, su rol aparece más relevante en las áreas de asistencia técnica, particularmente en lo relacionado con procesos productivos y control de calidad; la participación de las firmas clientes ha sido muy pobre y su mención como fuentes es muy limitada, concentrándose en algunas áreas de asistencia técnica.

\subsection{Dependencia de las empresas proveedoras de sus firmas clientes}

Pregunta 3: ¿Qué tipo de asistencia es requerida para el futuro y desde que actor se espera?

La información recogida frente al tema de cómo son las relaciones que se mantienen con las firmas clientes se presenta en el Gráfico 10.

En ella, se revela que el tipo de relación es mayoritariamente amigable e informativa (58\%), hecho que no deja de llamar la atención dada la pobreza de la asistencia reconocida a esta fuente particular.

\section{Gráfico 10}

Tipo de relación entre empresas proveedoras y firmas clientes

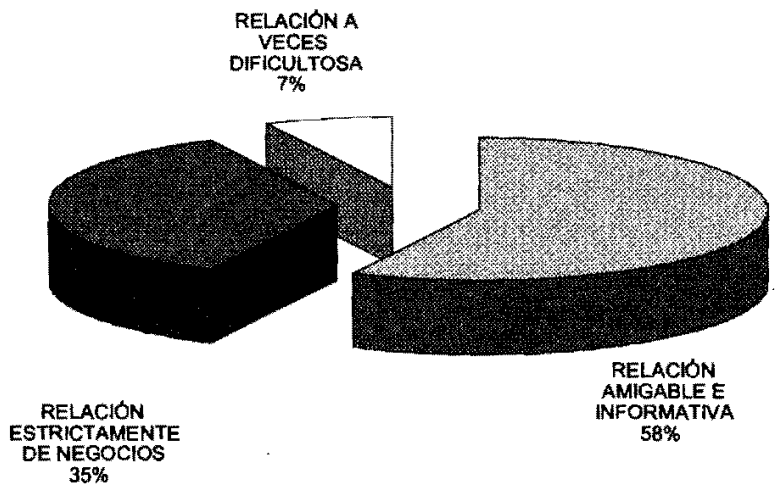

9 Sea: $r_{\text {tg }}^{h}=1$ si la firma i estima que el área de desarrollo $h$ ha contado con el apoyo o asistencia del actor o fuente particular $g . r_{\text {, }}^{h}=0$ si la firma l estima que no ha contado con apoyo.

La participación relativa de cada fuente de asistencia $g$ en un área $h$ específica es: $\gamma_{h}^{g}=\left\{\left.\frac{\sum_{j=1}^{1} r_{k}^{n}}{\mid \sum_{g=1}^{G} \sum_{k=1}^{1} r_{k g}^{n}}\right|^{n} \alpha_{h}\right.$, donde $l=136$ empresas proveedoras, $h=567$ según sean de asistencia tếcnica o de asistencia en gestión y $G=4$ fuentes. 


\section{Tabla $N^{2} 6$}

\section{Importancia de las áreas de asistencia}

\begin{tabular}{lr}
\hline Area de Asistencia Técnica & Futuro \\
Proceso Productivo y Control de Calidad & 0.29 \\
Provisión de Equipos Computacionales & 0.28 \\
Provisión de Herramientas y Maquinaria & 0.23 \\
Compra de Materia Prima & 0.18 \\
Otros Aspectos & 0.03 \\
Área de Asistencia de Gestión & \\
Contabilidad & 0.15 \\
Gerencia Administrativa y Personal & 0.18 \\
Marketing & 0.19 \\
Créditos a Plazos Convenientes & 0.19 \\
Préstamos No Reembolsables & 0.13 \\
Ayuda Financiera para I\&D & 0.16 \\
Otros & 0.00
\end{tabular}

Fuente: Elaboración propia.

Esta clase de relaciones configura un escenario de requerimientos futuros de asistencia técnica y de gestión que es similar al presente, saivo en las áreas de provisión de herramientas y maquinarias, y de compra de materia prima donde se aprecia un aumento leve. En el caso de la asistencia en gestión es notable la menor importancia futura del área contable, el fortalecimiento de los préstamos no reembolsables, y la ayuda financiera para investigación y desarrollo. Esta información está contenida en la Tabla № 6 .

Estas áreas emergentes en importancia, revelarían el esfuerzo por complejizar los servicios y productos ofrecidos a las firmas clientes. Esta intención sería consistente con la declaración de mejoramiento unilateral de la relación con los clientes (Gráfico 7) y con los cambios esperados en las estrategias de adquisiciones esperadas, más intensivas en la calidad y en su preocupación por el impacto sobre el medio ambiente (Gráfico 5 ).

Finalmente, las fuentes esperadas de apoyo para las diferentes áreas de asistencia se presentan en los Gráficos 11 y 12. Los dos gráficos ilustran, desde una perspectiva dinámica, la comparación de las áreas prioritarias de asistencia técnica y de gestión entre el presente y el futuro, pero distinguiendo como contribuyen a la variación las diferentes fuentes potenciales de asistencia ${ }^{10}$; to-

10 Dado que $y_{h}^{g}$ es un indicador que puede calcularse para dos periodos $t=1$ y $t=2$ se define un indicador de variación en la importancia de las áreas de asistencia (técnica o de gestión) según sea la fuente de apoyo con la siguiente forma: $\Delta_{h}^{g}=\left[\left(\gamma_{h t=2}^{g}-\gamma_{h t-1}^{g}\right) / \gamma_{h t-1}^{g}\right] \forall g=1 \ldots 4$. 


\section{Gráfico 11}

Variación en la importancia de las áreas de asistencia técnica según fuente de apoyo (comparación presente y futuro)

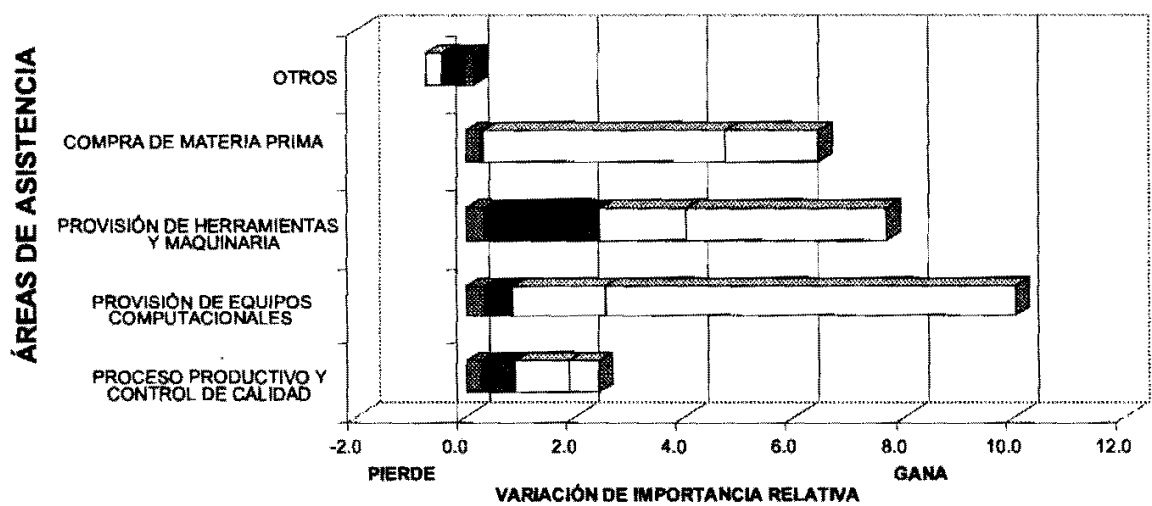

EMPRESAINTERNA ICONSULTOR PRNADO DEMPRESA MINERA CLIENTE DINSTITUCIONES DEAPOYO

\section{Gráfico 12}

Variación en la importancia de las áreas de asistencia de gestión según fuente de apoyo (comparación presente y futuro)

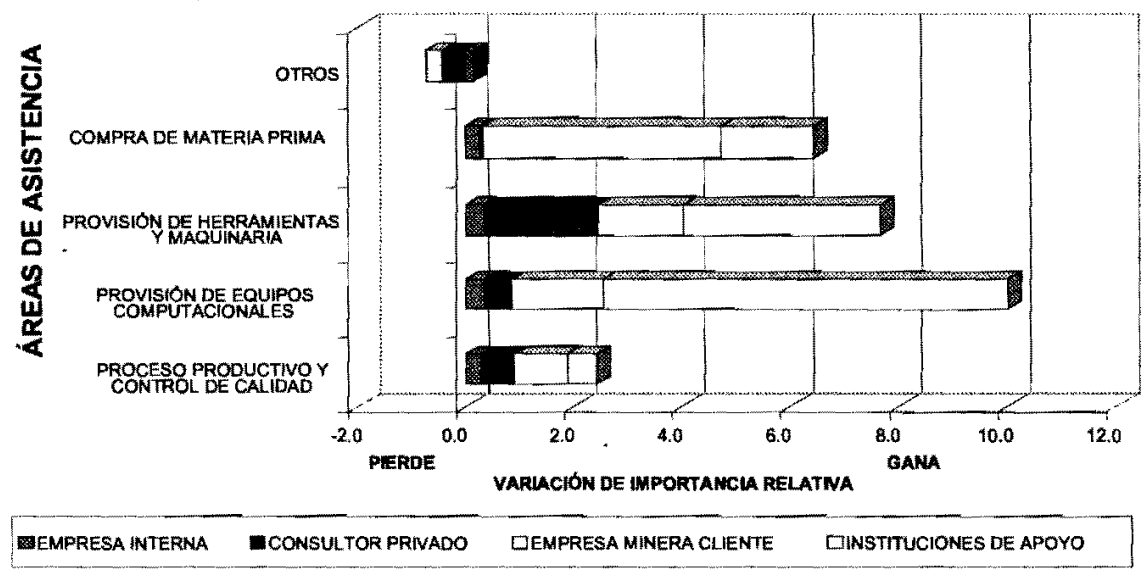

mando como referencia la situación presente registrada en el punto desarrollado con anterioridad.

En todas las áreas se aprecia un incremento de su importancia relativa, señal inequivoca del reconocimiento que las firmas hacen de un futuro más compe- titivo y tecnológicamente más complejo, pero las fuentes de contribución al proceso son diferenciadas. Debe notarse que en el análisis de la situación presente la fuente dominante de asistencia era la misma empresa, por lo que las ganancias positivas de otras fuentes en la situación 
esperada es significativa, por cuanto señala una mayor apertura de la firma para buscar y aceptar asistencia externa. En el caso particular de la asistencia técnica la mayor variación asociada a las empresas clientes es la adquisición de materia prima, donde se espera una asistencia notablemente mayor de ellas; por el contrario la provisión de herramientas y equipos, así como la de equipos computacionales contiene una mayor participación de las entidades de apoyo sectoriales.

En el caso de la asistencia en gestión, revela que la variaciones de asistencia más importantes para las firmas clientes corresponden a ayuda financiera en general, ya sea para investigación y desarrollo, y por mejores condiciones de reembolsos y plazos.

Para el caso de las instituciones de apoyo, las áreas anteriores también son importantes, pero destaca en forma relativa el apoyo en marketing y en gerencia administrativa y de personal.

Resulta muy interesante la creciente importancia que estaría cobrando la ayuda financiera para investigación y desarrollo, lo que sumado a las necesidades de equipos computacionales, podria estar asociado a la expectativa de una intensificación de las actividades de comercio y negocios electrónicos.

\section{Comentario final y conclusiones}

Ellram (1990) nota que la investigación en Selección de Proveedores como base de la estrategia SBM tiende a ser descriptiva o prescriptiva. Los estudios descriptivos proveen información sobre que cosas las firmas clientes están ha- ciendo para seleccionar a las firmas abastecedoras, mientras que los segundos enfatizan lo que "debería hacerse" en un sentido normativo.

Este estudio ha tratado de combinar ambos aspectos, describiendo cómo las estrategias de los compradores afectan a los proveedores y como la perspectiva de los proveedores debería ser incorporada en el diseño de la estrategia de abastecimiento de las firmas clientes.

La investigación empírica ha revelado importantes puntos sobre como la perspectiva de los proveedores puede contribuir al proceso de decisiones asociado a las estrategias de SBM. Si los compradores pudieran recibir o considerar este flujo de información, obtendrian visiones acerca de sus propias capacidades y de su dependencia de los proveedores. De aprovecharse esta perspectiva, sería posible que las firmas compradoras tomaran decisiones con mayor $y$ mejor información para elaborar sus diseños estratégicos de racionalización de su base de proveedores o "socios abastecedores". De este modo, la importancia de una comunicación e interacción regular entre compradores y vendedores puede ser vista no sólo desde una perspectiva de gestión, sino también desde la perspectiva de desarrollo, que recientemente se ha concedido a las redes de subcontratación industriales.

Finalmente, futuras investigaciones deberían considerar los aspectos de los costos que representan para las firmas clientes considerar la perspectiva y participación de los proveedores en las etapas iniciales del diseño de estrategias SBM, así como identificar cuáles son los beneficios financieros asociados a esta decisión. 


\section{Bibliografía Citada}

Auty, R.A. and Mikesell, Raymond F. (1998). Sustaining Development in Mineral Economies. Oxford, Clarendon.

Battat, J.; Frank, I. \& Shen, X. (1996). Suppliers to Multinationals- Linkage Programs to Strengthen Local Companies in Developing Countries. Occasional Paper No. 6 Foreign Investment Advisory Service Washington, D.C.: The World Bank.

Ellram, L (1990). The Supplier Selection Decision in Strategic Partnerships. International Journal of Purchasing and Materials Management No. 26 No.4 pp. 8-14.

Hahn, C.; Watts, C.A. \& Kim, K.Y. (1990). The Supplier Development Program: A Conceptual Model. International Journal of Purchasing and Materials Management. Vol. 26 No.2 pp. 2-25.

Halbach, A (1989). Multinational Enterprises and Subcontracting in the Third World: A Study of Inter-industrial Linkages. Working Paper No. 58 Multinational Enterprises Program, International Labour Office (ILO), Geneva.

Krause, D \& Handfield, R. (1999). Developing a World-Class Supply Base. Focus Study Center for Advanced Purchasing Studies, Arizona.

Monczka, R.M. \& Trent, R. (1991). Evolving Sourcing Strategies for the 1990s. International Journal of Physical Distribution and Logistics Management pp. 4-12.
Monczka, R.M.; Trent, R.J. \& Callahan, T.J. (1993). Supply Base Strategies to Maximize Supplier Petormance. International Journal of Physical Distribution and Logistics Management Vol 23, No. 2 pp. 42-54.

Nelson, $\mathrm{J},(1996)$. Business as Partners in Development - Creating Wealth for Countries, Companies and Communities. Executive Summary London: The Prince of Wales Business Leader Forum.

Richardson, P. (1991). World Class Mineral Operations. CRS Perspectives No. 36 Centre for Resource Studies, Queens University, Ontario pp 9-22.

Singer, H.W. (1950). The Distribution of Gains between Investing and Borrowing Countries. The American Economic Review Vol XI, May pp. 475-476.

United Nations Industrial Development Organization (UNIDO) (1999). Supporting Private Industry. Private Sector Development Branch - Investment Promotion and Institutional Capacity Building Division UNIDO: Vienna.

Watts, C and Hahn, C. (1993). Supplier Development Programs: An Empirical Analysis. International Journal of Purchasing and Materials Management Vol. 29 No. 2 pp. 11-27.

Yotopoulos, Pan and Nugent, Jeffrey B. "A Balanced -Growth version of the Linkage Hypothesis: A Test. Quarterly Journal of Economics Vol. 87, May pp. 157-171. 